United Nations Educational Scientific and Cultural Organization

and

International Atomic Energy Agency

THE ABDUS SALAM INTERNATIONAL CENTRE FOR THEORETICAL PHYSICS

\title{
EXPONENTIAL FORMULA FOR THE REACHABLE SETS OF QUANTUM STOCHASTIC DIFFERENTIAL INCLUSIONS
}

\author{
E.O. Ayoola \\ Department of Mathematics, University of Ibadan, \\ Ibadan, Federal Republic of Nigeria ${ }^{1}$ \\ and
}

The Abdus Salam International Centre for Theoretical Physics, Trieste, Italy.

\begin{abstract}
We establish an exponential formula for the reachable sets of quantum stochastic differential inclusions (QSDI) which are locally Lipschitzian with convex values. Our main results partially rely on an auxilliary result concerning the density, in the topology of the locally convex space of solutions, of the set of trajectories whose matrix elements are continuously differentiable. By applying the exponential formula, we obtain results concerning convergence of the discrete approximations of the reachable set of the QSDI. This extends similar results of Wolenski [19] for classical differential inclusions to the present noncommutative quantum setting.
\end{abstract}

MIRAMARE - TRIESTE

July 2001

\footnotetext{
${ }^{1}$ Permanent address. Email: uimath@mail.skannet.com
} 


\section{INTRODUCTION}

We continue our studies in [5] concerning the reachable sets (or attainability sets) of quantum stochastic differential inclusions given by

$$
\begin{aligned}
d X(t) & \in E(t, X(t)) d \wedge_{\pi}(t)+F(t, X(t)) d A_{f}(t)+G(t, X(t)) d A_{g}^{+}(t) \\
& +H(t, X(t)) d t, \quad \text { almost all } \quad t \in[0, T] \\
X(0) & =X_{0} .
\end{aligned}
$$

In $(1.1), E, F, G, H$ lie in $L_{l o c}^{2}([0, T] \times \tilde{\mathcal{A}})_{m v s}, X:[0, T] \rightarrow \tilde{\mathcal{A}}$ belongs to $L_{\text {loc }}^{2}(\tilde{\mathcal{A}})$ and $\wedge_{\pi}, A_{f}, A_{g}^{+}:[0, T] \rightarrow \tilde{\mathcal{A}}$ are the driving gauge, annihilation and creation processes. As usual $\tilde{\mathcal{A}}$ is the locally convex space of noncommutative stochastic processes whose topology is generated by the family of seminorms $\left\{\|x\|_{\eta \xi}=|\langle\eta, x \xi\rangle|: x \in \tilde{\mathcal{A}}, \quad \eta, \xi \in \mathbb{D} \underline{\otimes} \mathbb{E}\right\}$ (see [2-5, 8-10] for details).

For arbitrary $\eta, \xi \in \mathbb{D} \underline{\otimes} \mathbb{E}$, it is well known (see $[3,4,8]$ ) that (1.1) is equivalent to the first order initial value nonclassical inclusion given by

$$
\begin{gathered}
\frac{d}{d t}<\eta, X(t) \xi>\in P(t, X(t))(\eta, \xi) \\
X(0)=X_{0}, \text { almost all } t \in[0, T]
\end{gathered}
$$

where $(\eta, \xi) \rightarrow P(t, X(t))(\eta, \xi)$ is a multivalued sesquilinear form on $\mathbb{D} \underline{\otimes} \mathbb{E}$ with values in $\mathbb{C}$, the field of complex numbers. The explicit form of the map $P$ is presented in Section 2 below.

By adopting similar notations as in Wolenski [19], involving reachable sets and sets of trajectories of classical differential inclusions, our considerations in this paper mainly focus on the reachable set $R^{(T)}\left(X_{0}\right)$, which is defined by

$$
R^{(T)}\left(X_{0}\right)=\{\Phi(T): \Phi(\cdot) \text { solves }(1.2)\}
$$

For arbitrary $\eta, \xi \in \mathbb{D} \underline{\otimes} \mathbb{E}$, we associate with (1.3) the set

$$
R^{(T)}\left(X_{0}\right)(\eta, \xi):=\left\{<\eta, \Phi(T) \xi>: \Phi(T) \in R^{(T)}\left(X_{0}\right)\right\} .
$$

Similarly, the set of trajectories of (1.2) is defined by

$$
S^{(T)}\left(X_{0}\right):=\{\Phi(\cdot): \Phi(\cdot) \text { solves }(1.2)\}
$$

Again, associated with (1.5), we define $S^{(T)}\left(X_{0}\right)(\eta, \xi)$ by :

$$
S^{(T)}\left(X_{0}\right)(\eta, \xi):=\left\{<\eta, \Phi(\cdot) \xi>: \Phi(\cdot) \in S^{(T)}\left(X_{0}\right)\right\} .
$$

The main result of this paper is that the exponential formula

$$
R^{(T)}\left(X_{0}\right)(\eta, \xi)=\lim _{N \rightarrow \infty}\left(I+\frac{T}{N} P\right)^{N}\left(X_{0}\right)(\eta, \xi)
$$


holds subject to the map $(t, x) \rightarrow P(t, x)(\eta, \xi)$ being locally Lipschitzian with convex values and the stochastic processes are defined only on a simple Fock space. The power of $\left(I+\frac{T}{N} P\right)$ in (1.7) is that of composition of multivalued sesquilinear forms, defined in Section 4 and the limit is a set limit in the sense of Kuratowski. The identity multifunction $I: \tilde{\mathcal{A}} \rightarrow 2^{\tilde{\mathcal{A}}}$ takes $x \rightarrow\{x\}$.

An important consequence of formula (1.7) is that solutions of the inclusion (1.1) or (1.2) need not be invoked in order to determine the points in $R^{(T)}\left(X_{0}\right)(\eta, \xi)$. This situation is similar to what was obtained in the case of reachable sets for classical differential inclusions as explained in $[19]$.

Another important feature of Equation (1.7) concerns discretizations of quantum stochastic differential inclusion (1.2). Equation (1.7) implies that the set of all endpoints of the matrix elements of discrete trajectories of (1.2) converge to the entire reachable set $R^{(T)}\left(X_{0}\right)(\eta, \xi)$. Consequently, we obtain convergence results concerning discrete approximate reachable sets of $(1.2)$.

This work is partly motivated by the need to develop numerical analysis of quantum stochastic differential inclusions. As highlighted in [5], emphasis so far has been on numerical procedures for continuous quantum stochastic differential equations with high degree of differentiability of the matrix elements of solutions (see $[2,3,4]$ ). The numerical analysis of the discontinuous equations needs to be developed as well since a large number of quantum stochastic differential equations arising from applications are discontinuous but may be reformulated as regularized inclusions. Questions concerning estimations of the Hausdorff distance between the sets of solutions of (1.2) and the set of solutions of its discrete approximation will be considered in a forthcoming paper.

The plan for the rest of the paper is as follows: Section 2 contains preliminary notations and basic prerequisite results. In Section 3, we establish a result concerning approximation of trajectories of (1.2) by trajectories whose matrix elements are continuously differentiable. This extends the result of Wolenski [19] concerning approximations of solutions of classical differential inclusions by $C^{1}$ trajectories. The main results, concerning the exponential formula, is established in section 4. We formulate the discrete Euler approximations of the reachable set of (1.2). Finally, we show that the discrete reachable sets converge to the entire reachable set of $(1.2)$.

\section{PRELIMINARY RESULTS AND ASSUMPTIONS}

As in [2-5,8-10], we associate with the locally convex state space $\tilde{\mathcal{A}}$ of noncommutative stochastic processes the spaces $\operatorname{Ad}(\tilde{\mathcal{A}}), \quad \operatorname{Ad}(\tilde{\mathcal{A}})_{w a c}, \quad L_{l o c}^{p}(\tilde{\mathcal{A}}), L_{\gamma, l o c}^{\infty}\left(\mathbb{R}_{+}\right)$for a fixed Hilbert space $\gamma$ and for $0<p<\infty$.

If $A$ is a topological space, then $\operatorname{clos}(A)($ resp. $\operatorname{comp}(A))$ denotes the collection of nonvoid closed (resp. compact) subsets of $A$. 
We employ the Hausdorff topology $\tau_{H}$ on $\cos (\tilde{\mathcal{A}})$ determined by a family of pseudo-metrics $\left\{\rho_{\eta \xi}(\cdot), \eta, \xi \in \mathbb{D} \underline{\otimes} \mathbb{E}\right\}$ on $\operatorname{clos}(\tilde{\mathcal{A}})$ as follows:

For $x \in \tilde{\mathcal{A}}, \mathcal{M}, \mathcal{N} \in \operatorname{clos}(\tilde{\mathcal{A}})$

$$
\begin{aligned}
\mathbf{d}_{\eta \xi}(x, \mathcal{N}) & \equiv \inf _{y \in \mathcal{N}}\|x-y\|_{\eta \xi}, \\
\delta_{\eta \xi}(\mathcal{M}, \mathcal{N}) & \equiv \sup _{x \in \mathcal{M}} \mathbf{d}_{\eta \xi}(x, \mathcal{N}),
\end{aligned}
$$

and

$$
\rho_{\eta \xi}(\mathcal{M}, \mathcal{N}) \equiv \max \left(\delta_{\eta \xi}(\mathcal{M}, \mathcal{N}), \delta_{\eta \xi}(\mathcal{N}, \mathcal{M})\right)
$$

For $\mathcal{M} \in \operatorname{clos}(\tilde{\mathcal{A}}),\|\mathcal{M}\|_{\eta \xi}:=\mathbf{d}_{\eta \xi}(\mathcal{M},\{0\})$.

Similarly, for $A, B \in \operatorname{clos}(\mathscr{C})$ and $x \in \mathscr{C}$, the complex numbers, let

$$
\begin{aligned}
\mathbf{d}(x, A) & =\inf _{y \in A}|x-y| \\
\delta(A, B) & =\sup _{x \in A} \mathbf{d}(x, B)
\end{aligned}
$$

and

$$
\rho(A, B)=\max (\delta(A, B), \delta(B, A))
$$

Then we employ the metric topology on $\operatorname{clos}(\mathscr{C})$ induced by $\rho$. The set -theoretic operations are adopted as usual (see [8-10] for details).

In the following, Kuratowski limits of set will be frequently employed. If $\left\{\mathcal{M}_{j}\right\}_{j=1}^{\infty}$ is a sequence of subsets of $\tilde{\mathcal{A}}$, we define the limsup and liminf of $\left\{\mathcal{M}_{j=1}^{\infty}\right\}$ by

$$
\begin{aligned}
& \lim _{j \rightarrow \infty} \sup \mathcal{M}_{j}=\left\{a: \lim _{j \rightarrow \infty} \inf \mathbf{d}_{\eta \xi}\left(a, \mathcal{M}_{j}\right)=0\right\} \\
& \lim _{j \rightarrow \infty} \inf \mathcal{M}_{j}=\left\{a: \lim _{j \rightarrow \infty} \sup \mathbf{d}_{\eta \xi}\left(a, \mathcal{M}_{j}\right)=0\right\} .
\end{aligned}
$$

If $\lim \sup \mathcal{M}_{j}=\liminf \mathcal{M}_{j}$, we say that the limit exists and write $\lim _{j \infty} \mathcal{M}_{j}$ for the common value. We observe that if each $\mathcal{M}_{j}$ and $A$ are compact in $\tilde{\mathcal{A}}$ and contained in a bounded set, then from (2.1) and (2.2), $A=\lim _{j \rightarrow \infty} \mathcal{M}_{j}$ if and only if $\rho_{\eta \xi}\left(\mathcal{M}_{j}, A\right) \rightarrow 0$ as $j \rightarrow \infty$.

Similar definitions hold for the Kuratowski limit of a sequence of subsets of $\mathscr{C}$, the field of complex numbers. However, the Hausdorff metric $\rho$ will now replace the family of pseudo metric above.

\section{Continuous Multivalued Stochastic Processes}

A multivalued stochastic process indexed by the set $[0, T] \subseteq \mathbb{R}_{+}$is a multifunction on $[0, T]$ with values in $\cos (\tilde{\mathcal{A}})$. As in [5], the set of all locally p-integrable multivalued stochastic processes will be denoted by $L_{\text {loc }}^{P}(\tilde{\mathcal{A}})_{m v s}, p \in(0, \infty)$ while $L_{l o c}^{p}(I \times \tilde{\mathcal{A}})_{m v s}$ is the set of maps 
$\Phi: I \times \tilde{\mathcal{A}} \mapsto \operatorname{clos}(\tilde{\mathcal{A}})$ such that the map $t \rightarrow \Phi(t, X(t)), t \in[0, T]$ lies in $L_{\text {loc }}^{P}(\tilde{\mathcal{A}})_{m v s}$ for every $X \in L_{l o c}^{p}(\tilde{\mathcal{A}})$.

For $f, g \in L_{\gamma, l o c}^{\infty}\left(\mathbb{R}_{+}\right), \pi \in L_{B(\gamma), l o c}^{\infty}\left(\mathbb{R}_{+}\right), \mathbf{1}$ is the identity map on $\mathcal{R} \otimes \Gamma\left(L_{\gamma}^{2}\left(\mathbb{R}_{+}\right)\right)$and $\mathrm{M}$ is any of the processes $A_{f}, A_{g}^{+}, \wedge_{\pi}$ and $s \mapsto s \mathbf{1}, s \in \mathbb{R}_{+}$, then the multivalued stochastic integral $\int_{t_{0}}^{t} \Phi(s, X(s)) d M(s)$ is adopted as in [8].

Let $G:[0, T] \rightarrow 2^{\tilde{\mathcal{A}}}$ be a given multivalued stochastic process indexed by $[0, T]$. Then we say that $G$ is upper semicontinuous at $t_{0} \in[0, T]$ if

$$
\lim _{j \rightarrow \infty} \sup G\left(t_{j}\right) \subseteq G\left(t_{0}\right)
$$

for all sequences $\left\{t_{j}\right\}$ with $t_{j} \rightarrow t_{0}$.

The map $G$ is lower semicontinuous at $t_{0}$ if

$$
G\left(t_{0}\right) \subseteq \lim _{j \rightarrow \infty} \inf G\left(t_{j}\right)
$$

for all $\left\{t_{j}\right\}$ with $t_{j} \rightarrow t_{0}$.

$G$ is said to be continuous if it is both upper and lower semicontinuous. Similar definitions of continuity hold for a multifunction of the form: $\Phi: \tilde{\mathcal{A}} \rightarrow 2^{\tilde{\mathcal{A}}}$.

\section{Lipschitzian Multifunctions}

These are defined as follows:

(i) Let $\mathcal{N}$ be an open subset of $\tilde{\mathcal{A}}$. A map $\Phi: \mathcal{N} \rightarrow \operatorname{comp}(\tilde{\mathcal{A}})$ will be called Lipschitzian if for all $\eta, \xi \in \mathbb{D} \underline{\otimes} \mathbb{E}$, there exist positive numbers $K_{\eta \xi}$ such that

$$
\rho_{\eta \xi}(\Phi(x), \Phi(y)) \leq K_{\eta \xi}\|x-y\|_{\eta \xi}, \forall x, y \in \mathcal{N}
$$

We say that $\Phi$ is locally Lipschitzian if it is Lipschitzian on each compact subset of $\mathcal{N}$.

(ii) If $\Phi: \mathcal{N} \rightarrow 2^{\operatorname{sesq}(\mathbb{D} \underline{\otimes} \mathbb{E})}$, where $\operatorname{sesq}(\mathbb{D} \underline{\otimes} \mathbb{E})$ is the linear space of sesquilinear forms on $\mathbb{D} \underline{\otimes} \mathbb{E}$, then $\Phi$ is Lipschitzian if

$$
\rho(\Phi(x)(\eta, \xi), \Phi(y)(\eta, \xi)) \leq K_{\eta \xi}\|x-y\|_{\eta \xi}
$$

where $K_{\eta \xi}$ are positive real numbers.

We remark here that by Proposition (6.2) in [8], the map $(t, x) \rightarrow P(t, x)(\eta, \xi)$ appearing in 
(1.2) is Lipschitzian if the coefficients $E, F, G, H:[0, T] \times \tilde{\mathcal{A}} \rightarrow 2^{\tilde{\mathcal{A}}}$ in (1.1) are Lipschitzian.

For $E, F, G, H$ lying in $L_{\text {loc }}^{2}(I \times \tilde{\mathcal{A}})_{m v s},(1.1)$ is understood as an integral inclusion given by

$$
\begin{aligned}
X(t) & \in X_{0}+\int_{0}^{t}\left(E(s, X(s)) d \wedge_{\pi}(s)+F(s, X(s)) d A_{f}(s)\right. \\
& \left.+G(s, X(s)) d A_{g}^{+}(s)+H(s, X(s)) d s\right), t \in[0, T]
\end{aligned}
$$

with initial data $\left(t_{0}, X_{0}\right)$.

The explicit form of the map $P(t, x)(\eta, \xi)$ is given as follows: For $(t, x) \in \mathbb{R}_{+} \times \tilde{\mathcal{A}}, \eta, \xi \in \mathbb{D} \underline{\otimes} \mathbb{E}$, such that $\eta=c \otimes e(\alpha), \xi=d \otimes e(\beta), c, d \in \mathbb{D}, \alpha, \beta \in L_{\gamma, l o c}^{\infty}\left(\mathbb{R}_{+}\right)$, define the multifunction

$$
P_{\alpha \beta}:[0, T] \times \tilde{\mathcal{A}} \rightarrow 2^{\tilde{\mathcal{A}}}
$$

by

$$
P_{\alpha \beta}(t, x)=\mu_{\alpha \beta}(t) E(t, x)+\nu_{\beta}(t) F(t, x)+\sigma_{\alpha}(t) G(t, x)+H(t, x)
$$

where

$$
\begin{gathered}
\mu_{\alpha \beta}(t)=<\alpha(t), \pi(t) \beta(t)>_{\gamma} \\
\nu_{\beta}(t)=<f(t), \beta(t)>_{\gamma}
\end{gathered}
$$

and

$$
\sigma_{\alpha}(t)=<\alpha(t), g(t)>_{\gamma}
$$

This leads to the multifunction

$$
P:[0, T] \times \tilde{\mathcal{A}} \rightarrow 2^{\operatorname{sesq}(\mathbb{D} \underline{\otimes} \mathbb{E})}
$$

defined by

$$
P(t, x)(\eta, \xi):=<\eta, P_{\alpha \beta}(t, x) \xi>=\left\{<\eta, Z(t, x) \xi>: Z(t, x) \in P_{\alpha \beta}(t, x)\right\}
$$

As in [19], we shall introduce the notion of escape times. In what follows, unless otherwise indicated, we consider the autonomous version of (1.1) and (1.2).

Let $\mathcal{N} \subseteq \tilde{\mathcal{A}}$ be an open subset and $x_{0} \in \mathcal{N}$. Assume that $P(x)(\eta, \xi)$ has compact values and is locally Lipschitzian on $\mathcal{N}$. Then we define the escape time $\tilde{T}$ by

$$
\tilde{T}:=\sup \left\{T: c l \bigcup_{0 \leq t \leq T} R^{(t)}\left(x_{0}\right) \text { is compact in } \mathcal{N}\right\},
$$

where " $\mathrm{cl}$ " denotes the closure of the set. 
Next, we present a non-commutative generalization of the Fillipov existence theorem for inclusion (1.2) due to [8], in a form suitable for our purpose. To this end, for an arbitrary process $Z:[0, T] \rightarrow \tilde{\mathcal{A}}$ lying in $\operatorname{Ad}(\tilde{\mathcal{A}})_{\text {wac }}$, we define

$$
\sigma(Z):=\int_{0}^{T} \mathbf{d}\left(\frac{d}{d t}<\eta, Z(t) \xi>, P(Z(t))(\eta, \xi)\right) d t .
$$

Theorem 2.1. Assume that the following conditions hold:

(a) $Z: I \mapsto \tilde{\mathcal{A}}$ is an arbitrary process lying in $\operatorname{Ad}(\tilde{\mathcal{A}})_{\text {wac }}$ such that there exists positive functions $W_{\eta \xi}(t)$ satisfying

$$
\mathbf{d}\left(\frac{d}{d t}<\eta, Z(t) \xi>, P(Z(t))(\eta, \xi)\right) \leq W_{\eta \xi}(t) .
$$

(b) There exists $\theta>0$ and $\mathcal{N} \subseteq \tilde{\mathcal{A}}$ such that each of the maps $E, F, G, H$ is Lipschitzian from $\mathcal{N}$ to $\left(\operatorname{clos}(\tilde{\mathcal{A}}), \tau_{H}\right)$ and that

$$
Q_{Z, \theta}=\left\{x \in \tilde{\mathcal{A}}:\|x-Z(t)\|_{\eta \xi} \leq \theta, \forall \eta, \xi \in \mathbb{D} \underline{\otimes} \mathbb{E} \text { for some } t \in[0, T]\right\} \subseteq \mathcal{N} .
$$

(c) $K_{\eta \xi}>0$ are the Lipschitz constants for the map $P: \mathcal{N} \rightarrow 2^{\operatorname{ses} q(\mathbb{D} \otimes \mathbb{E})}$ on $\mathcal{N}$.

(d) For arbitrary $\eta, \xi \in \mathbb{D} \underline{\otimes} \mathbb{E}, t \in[0, T]$,

$$
E_{\eta \xi}(t)=e^{t K_{\eta \xi}} \int_{0}^{t} d s W_{\eta \xi}(s) .
$$

If in addition, $E, F, G, H$ are continuous from $\tilde{\mathcal{A}}$ to $\left(\operatorname{clos}(\tilde{\mathcal{A}}), \tau_{H}\right)$ and

$$
\int_{0}^{T} W_{\eta \xi}(t) d t<\theta e^{-K_{\eta \xi} T},
$$

then there exists a solution $\left.\Phi \in S^{(} T\right)(Z(0))$ of (1.2) satisfying

$$
\|\Phi(t)-Z(t)\|_{\eta \xi}<\sigma(Z) e^{K_{\eta \xi} T}, \quad t \in J
$$

and

$$
\left|\frac{d}{d t}<\eta, \Phi(t) \xi>-\frac{d}{d t}<\eta, Z(t) \xi>\right| \leq K_{\eta \xi} E_{\eta \xi}(t)+W_{\eta \xi}(t)
$$

for almost all $t \in J$ where

$$
J=\left\{t \in[0, T]: E_{\eta \xi}(t) \leq \sigma(Z) e^{K_{\eta \xi^{T}}} \leq \theta\right\}
$$

The next result is a useful lemma due to Wolenski [19].

Lemma 2.2. Suppose that $R, S, M_{1}, M_{2}, M_{N}$ are real constants satisfying

$$
M_{j+1}=R+S M_{j}, \text { for } j=1,2 \cdots N,
$$


then

$$
\begin{aligned}
M_{N} & =R\left(\frac{1-S^{N}}{1-S}\right)+S^{N} M_{0} \text { if } S \neq 1 \\
& =N R+M_{0} \quad \text { if } S=1
\end{aligned}
$$

\section{SOME DENSITY RESULTS}

In this section, we show that an arbitrary trajectory of the Inclusion (1.2) may be approximated in $\tilde{\mathcal{A}}$ by a trajectory whose matrix elements are of class $C^{1}[0, T]$, provided that the coefficients $E, F, G, H$ are locally Lipschitzian and the map $P(x)(\eta, \xi)$ has convex values in $\mathbb{C}$. This extends similar results in [19] to the present non-commutative quantum setting. In what follows, in this Section and Section 4, we consider the initial space $\mathcal{R} \equiv \mathbb{C}$. Consequently, $\mathbb{D} \underline{\otimes} \mathbb{E}=\mathbb{E}$ and $\mathcal{R} \otimes \Gamma\left(L_{\gamma}^{2}\left(\mathbb{R}_{+}\right)\right) \equiv \Gamma\left(L_{\gamma}^{2}\left(\mathbb{R}_{+}\right)\right)$.

Theorem 3.1. Suppose that the following conditions hold.

(i) $\mathcal{N}$ is an open subset of $\tilde{\mathcal{A}}$ and $P: \mathcal{N} \rightarrow 2^{\operatorname{sesq}(\mathbb{E})}$ is a multivalued sesquilinear form with nonempty, convex and compact values in $\mathbb{C}$.

(ii) The coefficients $E, F, G, H$ are locally Lipschitzian on $\mathcal{N}$.

(iii) $\Phi(\cdot) \in S^{(T)}\left(x_{0}\right)$ is a trajectory of $(1.2)$ with matrix element

$$
<\eta, \Phi(\cdot) \xi>:=\Phi_{\eta \xi}(\cdot) \in S^{(T)}\left(x_{0}\right)(\eta, \xi) .
$$

Then for each $\epsilon>0$, there exists $\tilde{\Phi}(\cdot) \in S^{(T)}\left(x_{0}\right)$ such that

$$
<\eta, \tilde{\Phi}(\cdot) \xi>:=\tilde{\Phi}_{\eta \xi}(\cdot) \in S^{(T)}\left(x_{0}\right)(\eta, \xi) \bigcap C^{1}[0, T]
$$

and

$$
\|\Phi(t)-\tilde{\Phi}(t)\|_{\eta \xi}<\epsilon
$$

We first establish the following Proposition which will be employed in the proof of Theorem 3.1.

Proposition 3.2. Let $\mathcal{N}$, the map $P$ and the coefficients $E, F, G, H$ be as in Theorem (3.1) and suppose that the following hold.

(i) $Y:[0, T] \rightarrow \tilde{\mathcal{A}}$ is an arbitrary process lying in $\operatorname{Ad}(\tilde{\mathcal{A}})_{\text {wac }}$ such that its matrix elements 
$Y_{\eta \xi}(\cdot):=<\eta, Y(\cdot) \xi>$ belong to $C^{1}[0, T]$ for each pair $\eta, \xi \in \mathbb{E}$.

(ii) There is a compact set $Q \subseteq \tilde{\mathcal{A}}$ and $\theta>0$ such that the set

$$
\left\{x:\|x-Y(t)\|_{\eta \xi} \leq \theta, \text { for some } 0 \leq t \leq T \quad \forall \eta, \xi \in \mathbb{E}\right\} \subseteq Q \subseteq \mathcal{N} .
$$

Let $K_{\eta \xi}$ be Lipschitz constants for the map $P$ on $Q$. Assume further that

$$
\sigma(Y)<\theta e^{-K_{\eta \xi} T}
$$

Then, there exists a trajectory $\tilde{Y}(\cdot) \in S^{(T)}(Y(0))$ with

$$
\tilde{Y}_{\eta \xi}(\cdot) \in S^{(T)}(Y(0))(\eta, \xi) \bigcap C^{1}[0, T]
$$

satisfying

$$
\|Y(t)-\tilde{Y}(t)\|_{\eta \xi}<\sigma(Y) e^{k_{\eta \xi} T}
$$

We require the following lemma for the proof of Proposion (3.2).

If $A \subseteq \mathbb{C}$ is a closed, convex set and $a \in \mathbb{C}$, we denote by $\operatorname{proj}(a, A)$, the unique element in $A$ closest to the point $a$.

Lemma 3.3. Suppose that $G:[0, T] \rightarrow 2^{\tilde{\mathcal{A}}}$ is a multivalued stochastic process such that the map $t \rightarrow G(t)(\eta, \xi)$ is a continuous multivalued sesquilinear form with nonempty, closed and convex values on $[0, T]$. Suppose further that $V:[0, T] \rightarrow \tilde{\mathcal{A}}$ is an adapted process such that the map $t \rightarrow<\eta, V(t) \xi>$ is continuous for each pair of $\eta, \xi \in \mathbb{E}$. Then the map

$$
t \rightarrow \operatorname{proj}(<\eta, V(t) \xi>, G(t)(\eta, \xi))
$$

is continuous on $[0, T]$.

Proof: The proof is an adaptation of the arguments in ( [19], Lemma 3.3 ) as follows: For each pair of $\eta, \xi$, set

$$
P_{\eta \xi}(t):=\operatorname{proj}(<\eta, V(t) \xi>, G(t)(\eta, \xi))
$$

Let $t_{0} \in[0, T]$ and $\left\{t_{j}\right\}_{j \geq 1} \subseteq[0, T]$ with $t_{j} \rightarrow t_{0}$ as $j \rightarrow \infty$.

Since $t \rightarrow G(t)(\eta, \xi)$ is continuous, the sequence $\left\{P_{\eta \xi}\left(t_{j}\right)\right\}$ is bounded and therefore has a convergent subsequence.

We assume that $P_{\eta \xi}\left(t_{j}\right) \rightarrow \tilde{p}_{\eta \xi}$ as $j \rightarrow \infty$ by passing to a subsequence if necessary but retaining the same notation. To conclude the proof, it is sufficient for us to show that

$$
P_{\eta \xi}\left(t_{0}\right)=\tilde{P}_{\eta \xi}
$$


Since $t \rightarrow G(t)(\eta, \xi)$ is upper semicontinuous at $t_{0}$, we have $\tilde{p}_{\eta \xi} \in G\left(t_{0}\right)(\eta, \xi)$, where $\tilde{p}_{\eta \xi}=<$ $\eta, \tilde{p} \xi>$, for some $\tilde{p} \in G\left(t_{0}\right) \subseteq \tilde{\mathcal{A}}$.

Again, since $t \rightarrow G(t)(\eta, \xi)$ is lower semicontinuous at $t_{0}$, there exists $q_{\eta \xi, j} \in G\left(t_{j}\right)(\eta, \xi)$ such that $q_{\eta \xi, j} \rightarrow P_{\eta \xi}\left(t_{0}\right)$.

Hence, we have

$$
\begin{aligned}
& \left|V_{\eta \xi}\left(t_{0}\right)-\tilde{p}_{\eta \xi}\right|=\lim _{j \rightarrow \infty}\left|V_{\eta \xi}\left(t_{j}\right)-P_{\eta \xi}\left(t_{j}\right)\right| \\
& \leq \lim _{j \rightarrow \infty}\left|V_{\eta \xi}\left(t_{j}\right)-q_{\eta \xi, j}\right| \text { by definition of } P_{\eta \xi}\left(t_{j}\right) \\
& =\left|V_{\eta \xi}\left(t_{0}\right)-P_{\eta \xi}\left(t_{0}\right)\right|,
\end{aligned}
$$

by continuity of $V_{\eta \xi}(\cdot)$ and the absolute value function $|\cdot|$.

But $P_{\eta \xi}\left(t_{0}\right)$ is the unique element in $G\left(t_{0}\right)(\eta, \xi)$ closest to $V_{\eta \xi}\left(t_{0}\right)$. Therefore, the last inequality implies that

$$
P_{\eta \xi}\left(t_{0}\right)=\tilde{p}_{\eta \xi}
$$

Proof of Proposition 3.2. The stochastic process $Y \in A d(\tilde{\mathcal{A}})_{\text {wac }}$ is given such that < $\eta, Y(\cdot) \xi>\in C^{1}[0, T]$ for each $\eta, \xi \in \mathbb{E}$ and satisfies

$$
\sigma(Y)<\theta e^{-K_{\eta \xi} T}
$$

By Lemma 3.3,

$$
t \rightarrow V_{\eta \xi, 0}:=\operatorname{proj}\left(\frac{d}{d t}<\eta, Y(t) \xi>, P(Y(t))(\eta, \xi)\right)
$$

is continuous on $[0, T]$.

Set

$$
Y_{\eta \xi, 1}=<\eta, Y(0) \xi>+\int_{0}^{t} V_{\eta \xi, 0}(s) d s
$$

Then,

$$
Y_{\eta \xi, 1}(\cdot) \in C^{1}[0, T]
$$

with

$$
\frac{d}{d t} Y_{\eta \xi, 1}(t)=V_{\eta \xi, 0}(t) \in P(Y(t))(\eta, \xi)
$$

by definition.

Since $Y_{\eta \xi, 1}(t)$ is a sesquilinear form on $[0, T]$, there exists a stochastic process $Y_{1}:[0, T] \rightarrow \tilde{\mathcal{A}}$ such that

$$
Y_{\eta \xi, 1}(t)=<\eta, Y_{1} \xi>
$$

Since

$$
\begin{aligned}
\left|Y_{\eta \xi, 1}(t)-Y_{\eta \xi}(t)\right| & =\left|\int_{0}^{t}\left(V_{\eta \xi, 0}(s)-\frac{d}{d s}<\eta, Y(s) \xi>\right) d s\right| \\
& \leq \int_{0}^{t}\left|V_{\eta \xi, 0}(s)-\frac{d}{d s}<\eta, Y(s) \xi>\right| d s \\
& =\int_{0}^{t} \mathbf{d}\left(\frac{d}{d s}<\eta, Y(s) \xi>, P(Y(s))(\eta, \xi)\right) d s \\
& \leq \sigma(Y)<\theta .
\end{aligned}
$$


i.e.

$$
\left\|Y_{1}(t)-Y(t)\right\|_{\eta \xi}<\theta
$$

Then,

$$
Y_{1}(t) \in Q \subseteq \mathcal{N}, \forall t \in[0, T]
$$

Next, we set

$$
Y_{\eta \xi, 0}(\cdot)=Y_{\eta \xi}(\cdot)
$$

Inductively, suppose that $n \geq 1$ and that adapted processes $\left\{Y_{j}\right\}_{j=1}^{n}$ have been chosen such that the sequence $\left\{\left\langle\eta, Y_{j}(\cdot) \xi\right\rangle\right\}_{j=1}^{n}$ are continuously differentiable on $[0, T]$ for all $\eta, \xi \in \mathbb{E}$ satisfying (3.3) -(3.6) below, for all $0 \leq t \leq T$ and $j=1,2 \cdots n$

$$
\begin{gathered}
\frac{d}{d t}<\eta, Y_{j}(t) \xi>\in P\left(Y_{j-1}(t)\right)(\eta, \xi) \\
\left|\frac{d}{d t}<\eta, Y_{j}(t) \xi>-\frac{d}{d t}<\eta, Y_{j-1}(t) \xi>\right| \leq \frac{\sigma(Y) K_{\eta \xi}^{j-1} t^{j-2}}{(j-2) !}, \\
\left|<\eta, Y_{j}(t) \xi>-<\eta, Y_{j-1}(t) \xi>\right| \leq \sigma(Y) \frac{\left(K_{\eta \xi} t\right)^{j-1}}{(j-1) !} \\
Y_{j}(t) \in Q .
\end{gathered}
$$

First, we observe that when $j=1,(3.5)$ and (3.6) follow directly from (3.2), (3.3) is obvious and (3.4) is vacuous.

Next, we begin by defining

$$
V_{\eta \xi, n+1}(t)=\operatorname{proj}\left(\frac{d}{d t}<\eta, Y_{n}(t) \xi>, P\left(Y_{n}(t)\right)(\eta, \xi)\right)
$$

and

$$
\begin{aligned}
Y_{\eta \xi, n+1}(t)= & <\eta, Y(0) \xi>+\int_{0}^{t} V_{\eta \xi, n+1}(s) d s \\
& =<\eta, Y_{n+1}(t) \xi>
\end{aligned}
$$

for some stochastic process $Y_{n+1}:[0, T] \rightarrow \tilde{\mathcal{A}}$.

By Lemma 3.3, $t \rightarrow V_{\eta \xi, n+1}(t)$ is continuous and

$$
\frac{d}{d t} Y_{\eta \xi, n+1}(t)=V_{\eta \xi, n+1}(t) .
$$

This implies that

$$
Y_{\eta \xi, n+1}(\cdot) \in C^{1}[0, T] .
$$

Let $t \in[0, T]$, then

$$
\frac{d}{d t} Y_{\eta \xi, n+1}(t) \in P\left(Y_{n}(t)\right)(\eta, \xi)
$$


Therefore,

$$
\begin{aligned}
& \left|\frac{d}{d t} Y_{\eta \xi, n+1}(t)-\frac{d}{d t}<\eta, Y_{n}(t) \xi>\right| \\
& =\mathbf{d}\left(\frac{d}{d t}<\eta, Y_{n}(t) \xi>, P\left(Y_{n}(t)\right)(\eta, \xi)\right) \\
& \leq \rho\left(P\left(Y_{n-1}(t)\right)(\eta, \xi), P\left(Y_{n}(t)\right)(\eta, \xi)\right) \text { by }(3.3), \\
& \leq K_{\eta \xi}\left\|Y_{n-1}(t)-Y_{n}(t)\right\|_{\eta \xi} \text { by Lipschitz property, } \\
& =K_{\eta \xi}\left|<\eta, Y_{n-1}(t) \xi>-<\eta, Y_{n}(t) \xi>\right| \\
& \leq \sigma(Y) \frac{K_{\eta \xi}^{n} t^{n-1}}{(n-1) !} \text { by }(3.5) .
\end{aligned}
$$

The last inequality shows that (3.4) holds for $j=n+1$.

Next, we have

$$
\begin{aligned}
& \mid<\eta, Y_{n+1}(t) \xi>-<\eta, Y_{n}(t) \xi> \\
& \leq \int_{0}^{t}\left|\frac{d}{d s} Y_{\eta \xi, n+1}(s)-\frac{d}{d s}<\eta, Y_{n}(s) \xi>\right| d s \\
& \leq \sigma(Y) \int_{0}^{t} \frac{K_{\eta \xi}^{n} s^{n-1}}{(n-1) !} d s \text { by }(3.7) \\
& =\sigma(Y) \frac{\left(K_{\eta \xi} t\right)^{n}}{n !} .
\end{aligned}
$$

The last inequality shows that (3.5) holds for $j=n+1$. Finally, we have

$$
\begin{aligned}
& \left\|Y_{n+1}(t)-Y(t)\right\|_{\eta \xi} \\
& =\left|<\eta, Y_{n+1}(t) \xi>-<\eta, Y(t) \xi>\right| \\
& \leq \sum_{j=0}^{n}\left|<\eta, Y_{j+1}(t) \xi>-<\eta, Y_{j}(t) \xi>\right| \\
& \leq \sigma(Y) \sum_{j=0}^{n} \frac{\left(K_{\eta \xi} t\right)^{j}}{j !} \text { by }(3.5) \text { and }(3.8) \\
& \leq \sigma(Y) e^{K_{\eta \xi} T}<\theta .
\end{aligned}
$$

This shows that $Y_{n+1}(t) \in Q$. The induction proof is complete.

The foregoings imply the existence of a subsequence $\left\{Y_{j}\right\}_{j=1}^{\infty}$ of adapted processes $Y_{j}:[0, T] \rightarrow \tilde{\mathcal{A}}$ lying in $Q$ with the property that the sequence $\left\{\left\langle\eta, Y_{j}(\cdot) \xi\right\rangle\right\}_{j=1}^{\infty}$ are continuously differentiable on $[0, T]$.

It follows from (3.5) and (3.4) that $\left\{Y_{j}(t)\right\}$ is Cauchy in $Q$ with the property that $\left\{\frac{d}{d t}<\right.$ $\left.\eta, Y_{j}(t) \xi>\right\}$ is also Cauchy in $\mathbb{C}$, the field of complex numbers.

Consequently, $\left\{Y_{j}(t)\right\}$ converges uniformly to some $\tilde{Y}(t)$ in $Q$, i.e.

$$
\left\|Y_{j}(t)-\tilde{Y}(t)\right\|_{\eta \xi}=\left|<\eta, Y_{j}(t) \xi>-<\eta, \tilde{Y}(t) \xi>\right| \rightarrow 0 \text { as } j \rightarrow \infty .
$$

This implies that

$$
<\eta, Y_{j}(t) \xi>\rightarrow<\eta, \tilde{Y}(t) \xi>\text { as } j \rightarrow \infty
$$


The function $\left\langle\eta, \tilde{Y}(\cdot) \xi>\right.$ is continuously differentiable on $[0, \mathrm{~T}]$ since $\left\langle\eta, Y_{j}(\cdot) \xi>\right.$ lies in $C^{1}[0, T]$ for each $j$.

Hence

$$
\frac{d}{d t}<\eta, Y_{j}(t) \xi>\rightarrow \frac{d}{d t}<\eta, \tilde{Y}(t) \xi>
$$

By (3.4), $\frac{d}{d t}<\eta, \tilde{Y}(t) \xi>$ is continuous by the continuity of the sequence $\left\{\frac{d}{d t}<\eta, Y_{j}(t) \xi>\right\}$ on $[0, \mathrm{~T}]$ for each $j$.

Moreover, for each $t \in[0, T]$ and by (3.3), we have

$$
\begin{aligned}
\frac{d}{d t}<\eta, \tilde{Y}(t) \xi> & =\lim _{j \rightarrow \infty} \frac{d}{d t}<\eta, Y_{j}(t) \xi> \\
& \in \lim _{j \rightarrow \infty} P\left(Y_{j-1}(t)\right)(\eta, \xi) \\
& =P(\tilde{Y}(t))(\eta, \xi) .
\end{aligned}
$$

Hence,

$$
\tilde{Y}(\cdot) \in S^{(T)}(Y(0)
$$

with

$$
<\eta, \tilde{Y}(\cdot) \xi>\in S^{(T)}(Y(0))(\eta, \xi)
$$

Finally, from (3.9), we obtain

$$
\begin{aligned}
&\|\tilde{Y}(t)-Y(t)\|_{\eta \xi}=\lim _{n \rightarrow \infty}\left\|Y_{n+1}(t)-Y(t)\right\|_{\eta \xi} \\
& \leq \sigma(Y) e^{K_{\eta \xi} T}
\end{aligned}
$$

The next result is a direct application of Lusin's Theorem (see Rudin [16], Phillips [15]) to elements of $A d(\tilde{\mathcal{A}})$ with Lebesque measurable matrix elements. The result will be employed in the proof of Theorem (3.1) that follows.

Theorem 3.4: Assume that the following conditions hold:

$f:[0, T] \rightarrow \tilde{\mathcal{A}}$ is a stochastic process such that for all $\eta, \xi \in \mathbb{E}$,

$$
f_{\eta \xi}(t):=<\eta, f(t) \xi>
$$

is Lebesque measurable on $[0, T]$ and

$$
R_{\eta \xi}=\sup _{[0, T]}\left|f_{\eta \xi}(t)\right| .
$$

Then, given $\epsilon>0$, there exists a borel subset $J \subseteq[0, T]$ and a continuous function $Z_{\eta \xi}:[0, T] \rightarrow$ $\mathbb{C}$ such that

$$
Z_{\eta \xi}(t)=f_{\eta \xi}(t), \quad t \in[0, T]-J
$$




$$
\sup _{[0, T]}\left|Z_{\eta \xi}(t)\right| \leq R_{\eta \xi}
$$

and

$$
L(J)<\epsilon,
$$

where $L$ denotes the Lebesque measure.

Proof: Since $f_{\eta \xi}(t)$ is measurable on $[0, T]$ and $L([0, T])<\infty$, then, by Lusin's Theorem, (see Phillip [15] page 225 ) there exists a Borel subset $J \subseteq[0, T]$ such that

$$
L(J)<\epsilon
$$

and

$$
f_{\eta \xi}(\cdot) \in C([0, T]-J)
$$

Next we define

$$
\begin{gathered}
Z_{\eta \xi}(t)=f_{\eta \xi}(t), \quad t \in[0, T]-J, \\
Z_{\eta \xi}(t)=\frac{R_{\eta \xi}}{|<\eta, \xi>|}<\eta, \xi>, \quad t \in J .
\end{gathered}
$$

We note that $Z_{\eta \xi}(\cdot)$ is well defined since $|\langle\eta, \xi\rangle| \neq 0, \forall \eta, \xi \in \mathbb{E}$, where

$$
\begin{gathered}
<\eta, \xi>=e^{<\alpha, \beta>}, \\
\eta=e(\alpha), \xi=e(\beta), \alpha, \beta \in L_{\gamma}^{2}\left(\mathbb{R}_{+}\right) .
\end{gathered}
$$

Consequently,

$$
Z_{\eta \xi}(\cdot) \in C[0, T]
$$

and the set

$$
J=\left\{t \in[0, T]: Z_{\eta \xi}(t) \neq f_{\eta \xi}(t)\right\}
$$

satisfies

$$
L(J)<\epsilon .
$$

Again,

$$
\sup _{[0, T]}\left|Z_{\eta \xi}(t)\right| \leq \sup _{[0, T]}\left|f_{\eta \xi}(t)\right|=R_{\eta \xi}
$$

Proof of Theorem 3.1: Given that $\Phi(\cdot) \in S^{(T)}\left(X_{0}\right)$ and $\epsilon>0$, we have $\Phi_{\eta \xi}(\cdot) \in S^{(T)}\left(X_{0}\right)(\eta, \xi)$ for each pair of $\eta, \xi \in \mathbb{E}$. We show that there exists a trajectory $\tilde{\Phi}(\cdot)$ such that

$$
\tilde{\Phi}_{\eta \xi}(\cdot) \in S^{(T)}\left(X_{0}\right)(\eta, \xi) \bigcap C^{1}[0, T]
$$

and

$$
\|\Phi(t)-\tilde{\Phi}(t)\|_{\eta \xi}<\epsilon,
$$


where

$$
\tilde{\Phi}_{\eta \xi}(\cdot)=<\eta, \tilde{\Phi}(\cdot) \xi>
$$

We assume without loss of generality that $\epsilon$ is sufficiently small so that the following hold

$$
\left\{u:\|u-\Phi(t)\|_{\eta \xi} \leq \epsilon, \text { for some } 0 \leq t \leq T, \forall \eta, \xi \in \mathbb{E}\right\} \subseteq Q \subseteq \mathcal{N},
$$

for some compact set $Q$ contained in $\mathcal{N}$.

Let $K_{\eta \xi} \geq 1$ be Lipschitz constants for the map $x \rightarrow P(x)(\eta, \xi)$ on $Q$ and let

$$
R_{\eta \xi}=\sup \left\{\left|v_{\eta \xi}\right|: v_{\eta \xi} \in \bigcup_{u \in Q} P(u)(\eta, \xi)\right\}
$$

Since $\Phi(\cdot) \in S^{(T)}\left(X_{0}\right)$, we have

$$
\left|\frac{d}{d t}<\eta, \Phi(t) \xi>\right| \leq R_{\eta \xi},
$$

for almost all $\mathrm{t}$ satisfying $0 \leq t \leq T$.

Let

$$
f_{\eta \xi}(t)=\frac{d}{d t}<\eta, \Phi(t) \xi>, \quad t \in[0, T],
$$

then by Theorem (3.3), there exists continuous functions $Z_{\eta \xi}(\cdot)$ on $[0, T]$ and a Borel subset $J \subseteq[0, T]$ such that

$$
\begin{gathered}
Z_{\eta \xi}(t)=f_{\eta \xi}(t), \text { for } t \in[0, T]-J, \forall \eta, \xi, \\
\sup _{[0, T]}\left|Z_{\eta \xi}(t)\right| \leq R_{\eta \xi},
\end{gathered}
$$

and

$$
L(J) \leq \frac{\epsilon}{4 K_{\eta \xi} R_{\eta \xi}(1+T) e^{K_{\eta \xi} T}},
$$

where $L$ is the Lebesque measure on $[0, T]$.

Next we define for each pair of $\eta, \xi$

$$
Y_{\eta \xi}(t)=<\eta, X_{0} \xi>+\int_{0}^{t} Z_{\eta \xi}(s) d s
$$

Then

$$
Y_{\eta \xi}(\cdot) \in C^{1}[0, T]
$$

As $Y_{\eta \xi}(t)$ is a sesquilinear form, there exists a stochastic process $Y:[0, T] \rightarrow \tilde{\mathcal{A}}$ such that

$$
Y_{\eta \xi}(t)=<\eta, Y(t) \xi>\text {, almost all } t \in[0, T]
$$

It is immediate that $Y$ lies in $\operatorname{Ad}(\tilde{\mathcal{A}})_{\text {wac }}$ by definition. 
Observe that for all $t \in[0, T]-J,<\eta, Y(t) \xi>-<\eta, \Phi(t) \xi>=0$.

However, for all $t \in[0, T]$, the following hold:

$$
\begin{aligned}
& |<\eta, Y(t) \xi>-<\eta, \Phi(t) \xi>|=\|Y(t)-\Phi(t)\|_{\eta \xi} \\
& \leq \int_{J}\left|Z_{\eta \xi}(s)-\frac{d}{d s}<\eta, \Phi(s) \xi>\right| d s \\
& \leq 2 R_{\eta \xi} L(J) \\
& \leq \frac{\epsilon}{2 K_{\eta \xi} e^{K_{\eta \xi} T}(1+T)} \\
& \leq \frac{\epsilon}{2}
\end{aligned}
$$

Again, we observe that (3.10) implies that the set $\left\{u:\|u-Y(t)\|_{\eta \xi} \leq \frac{\epsilon}{2}\right\}$ is contained in $Q$. Next, we estimate $\sigma(Y)$ as follows:

$$
\begin{aligned}
\sigma(Y) & :=\int_{0}^{T} \mathbf{d}\left(\frac{d}{d t}<\eta, Y(t) \xi>, P(Y(t))(\eta, \xi)\right) \\
\leq & \int_{[0, T]-J} \rho(P(\Phi(t))(\eta, \xi), P(Y(t))(\eta, \xi)) d t \\
& +\int_{J} \mathbf{d}\left(\frac{d}{d t}<\eta, Y(t) \xi>, P(Y(t))(\eta, \xi)\right) d t \\
\leq & K_{\eta \xi} \int_{0}^{T}\|\Phi(t)-Y(t)\|_{\eta \xi} d t+2 R_{\eta \xi} L(J) .
\end{aligned}
$$

Consequently, by applying (3.10), we have

$$
\begin{gathered}
\sigma(Y) \leq \frac{K_{\eta \xi} T \epsilon}{2 K_{\eta \xi} e^{K_{\eta \xi} T}(1+T)}+\frac{\epsilon}{2 K_{\eta \xi} e^{K_{\eta \xi} T}(1+T)} \\
=\frac{\epsilon}{2}\left[\frac{K_{\eta \xi} T+1}{K_{\eta \xi}(1+T)}\right] e^{-K_{\eta \xi} T} \\
<\frac{\epsilon}{2} e^{-K_{\eta \xi} T}, \text { since } K_{\eta \xi} \geq 1 .
\end{gathered}
$$

Application of Proposition (3.2) to the process $Y:[0, T] \rightarrow \tilde{\mathcal{A}}$ with $\theta=\frac{\epsilon}{2}$ and $Q$, implies that there exists $\tilde{\Phi}(\cdot) \in S^{(T)}\left(X_{0}\right)$ such that

$$
\tilde{\Phi}_{\eta \xi}(\cdot) \in S^{(T)}\left(X_{0}\right)(\eta, \xi) \bigcap C^{1}[0, T] \forall \eta, \quad \xi \in \mathbb{E}
$$

and

$$
\|Y(t)-\tilde{\Phi}(t)\|_{\eta \xi}<\frac{\epsilon}{2}
$$

Finally, by employing (3.10) again, we conclude that

$$
\|\Phi(t)-\tilde{\Phi}(t)\|_{\eta \xi} \leq\|\Phi(t)-Y(t)\|_{\eta \xi}+\|Y(t)-\tilde{\Phi}(t)\|_{\eta \xi}<\epsilon .
$$

i.e

$$
\|\Phi(t)-\tilde{\Phi}(t)\|_{\eta \xi}<\epsilon .
$$




\section{THE EXPONENTIAL FORMULA}

We first present the definitions of composition of multifunctions suitable for our purpose. Unless otherwise indicated, $\eta, \xi \in \mathbb{E}$ such that $\eta=e(\alpha), \quad \xi=e(\beta), \quad \alpha, \beta \in L_{\gamma}^{2}\left(\mathbb{R}_{+}\right)$. In what follows $I$ is the multifunction that takes $x \rightarrow\{x\}$.

Definition 4.1. Let $G_{0}$ and $G_{1}: \tilde{\mathcal{A}} \rightarrow 2^{\tilde{\mathcal{A}}}$ be multifunctions defined on $\tilde{\mathcal{A}}$. By composition $G_{0} \circ G_{1}: \tilde{\mathcal{A}} \rightarrow 2^{\tilde{\mathcal{A}}}$ of $G_{1}$ with $G_{0}$, we mean the set

$$
G_{0} \circ G_{1}(x)=\left\{z: \text { there exists } y \in G_{1}(x) \text { with } z \in G_{0}(y)\right\} \text {. }
$$

$G_{0}^{N}$ denotes the composition of $G_{0}$ with itself $N$ times.

Definition 4.2: The composition of the multivalued sesquilinear form $P: \tilde{\mathcal{A}} \rightarrow 2^{\text {sesq(}(\mathbb{E})}$ with itself $N$ times is defined by

$$
P^{N}(x)(\eta, \xi)=<\eta, P_{\alpha \beta}^{N}(x) \xi>,
$$

where

$$
P_{\alpha \beta}^{N}: \tilde{\mathcal{A}} \rightarrow 2^{\tilde{\mathcal{A}}}
$$

is the composition of $P_{\alpha \beta}$ with itself $N$ times in the sense of Definition (4.1).

Theorem 4.3: Suppose $\mathcal{N} \subseteq \tilde{\mathcal{A}}$ is open and $P: \tilde{\mathcal{A}} \rightarrow 2^{\text {sesq }(E)}$ is a locally Lipschitzian multivalued sequilinear form with nonempty, compact values on $\mathcal{N}$. Let $X_{0} \in \mathcal{N}$ be fixed.

(i) For $0 \leq T \leq \tilde{T}$, one has

$$
\lim _{N \rightarrow \infty} \sup \left(I+\frac{T}{N}\right)^{N}\left(X_{0}\right)(\eta, \xi) \subseteq c l R^{(T)}\left(X_{0}\right)(\eta, \xi)
$$

(ii) If in addition, $P$ is assumed to have convex values, then for all $T \geq 0$, we have

$$
R^{(T)}\left(X_{0}\right)(\eta, \xi) \subseteq \lim _{N \rightarrow \infty} \inf \left(I+\frac{T}{N} P\right)^{N}\left(X_{0}\right)(\eta, \xi) .
$$

Proof: (i) Suppose $0 \leq T \leq \tilde{T}$. Let $Q=c l \bigcup_{0 \leq t \leq T} R^{(t)}\left(X_{0}\right)$.

Then $Q$ is compact by definition of $\tilde{T}$. So there exists $\theta>0$ such that $Q+\theta B \subseteq \mathcal{N}$, where $B$ is the closed unit ball in $\tilde{\mathcal{A}}$.

We put

$$
\begin{aligned}
& R_{\eta \xi}=\sup \left\{\left|V_{\eta \xi}\right|: V_{\eta \xi} \in P(Q+\theta B)(\eta, \xi)\right\} \\
& :=\sup \left\{\left|V_{\eta \xi}\right|: V_{\eta \xi} \in \bigcup_{u \in Q+\theta B} P(u)(\eta, \xi)\right\}
\end{aligned}
$$


and put $K_{\eta \xi}>0$ to be Lipschitz constants for $P$ on $Q+\theta B$.

Let $\epsilon>0$. We show that for all large $N$ satisfying

$$
\frac{T}{N} \leq \min \left\{\frac{\epsilon}{R_{\eta \xi} K_{\eta \xi} T e^{K_{\eta \xi} T}}, \frac{\theta}{2 R_{\eta \xi}}\right\},
$$

the inclusion

$$
\left(I+\frac{T}{N} P_{\alpha \beta}\right)^{j}\left(X_{0}\right) \subseteq R^{\left(j \frac{T}{N}\right)}\left(X_{0}\right)+\epsilon B
$$

holds. Consequently, the inclusion

$$
\left(I+\frac{T}{N} P\right)^{j}\left(X_{0}\right)(\eta, \xi) \subseteq R^{\left(j \frac{T}{N}\right)}\left(X_{0}\right)(\eta, \xi)+\epsilon B(\eta, \xi)
$$

immediately follows from (4.4). Here, $B(\eta, \xi)=\{\langle\eta, x \xi\rangle: x \in B\}$. Since $\epsilon$ is arbitrarily small, we can then conclude that (4.1) holds.

In the sequel, we put $h=\frac{T}{N}, t_{j}=j h$ for $j=0,1,2 \cdots N$, where $N$ satisfies (4.3).

We shall establish (4.4) by induction on $j$. The case $j=0$ is trivial. For the induction hypothesis, suppose (4.4) holds for all $i$ such that $0 \leq i \leq j<N$.

Let $Y_{j+1} \in\left(I+h P_{\alpha \beta}\right)^{j+1}\left(X_{0}\right)$. Then there exists $Y_{0}=X_{0}, Y_{1}, Y_{2} \cdots Y_{j}$ and $U_{0}, U_{1} \cdots U_{j}$ so that for $0 \leq i \leq j$, we have

$$
U_{i} \in P_{\alpha \beta}\left(Y_{i}\right) \text { and } Y_{i+1}=Y_{i}+h U_{i}
$$

We remark that when $0 \leq i \leq j,(4.4)$ implies that

$$
Y_{i} \in Q+\frac{\theta}{2} B
$$

so that

$$
<\eta, U_{i} \xi>\in P(Q+\theta B)(\eta, \xi)
$$

and so

$$
\left|<\eta, U_{i} \xi>\right|=\left\|U_{i}\right\|_{\eta \xi} \leq R_{\eta \xi}
$$

Let $\Phi(\cdot)$ be defined on $\left[0, t_{j+1}\right]$ as the piecewise linear interpolation of $\left\{Y_{i}\right\}_{i=0}^{j+1}$ equally spaced on $\left[0, t_{j+1}\right]$ as follows

$$
\Phi(t)=Y_{i}+\left(t-t_{i}\right) U_{i} \text { if } t_{i} \leq t \leq t_{i+1} .
$$

$\Phi(t)$ is adapted and weakly absolutely continuous on $\left[0, t_{j+1}\right]$.

The range of $\Phi(\cdot)$ lies within $Q+\theta B$ because

$$
Y_{i}+\left(t-t_{i}\right) U_{i} \in Q+\frac{\theta}{2} B+h R_{\eta \xi} B \subseteq Q+\theta B .
$$

This follows from (4.3) since $h R_{\eta \xi} \leq \frac{\theta}{2}$.

Hence we have

$$
\sigma(\Phi)=\int_{0}^{t_{j+1}} \mathbf{d}\left(\frac{d}{d t}<\eta, \Phi(t) \xi>, P(\Phi(t))(\eta, \xi)\right) d t
$$




$$
\begin{aligned}
& \leq \sum_{i=0}^{j} \int_{t_{i}}^{t_{i+1}} \rho\left(P\left(Y_{i}\right)(\eta, \xi), P(\Phi(t))(\eta, \xi)\right) d t \\
& \leq K_{\eta \xi} \sum_{i=0}^{j} \int_{t_{i}}^{t_{i+1}}\left\|Y_{i}-\Phi(t)\right\|_{\eta \xi} d t \text { by Lipschitz property of } \mathrm{P} \\
& \leq K_{\eta \xi} T R_{\eta \xi} h \leq \epsilon e^{-K_{\eta \xi} T} \text { by }(4.3) .
\end{aligned}
$$

By Theorem (2.1), there exists a solution $\tilde{\Phi}$ of (1.2) such that

$$
\|\tilde{\Phi}(t)-\Phi(t)\|_{\eta \xi} \leq \sigma(\Phi) e^{K_{\eta \xi} T}, \quad 0 \leq t \leq t_{j+1}
$$

In particular, if we put $t=t_{j+1}, i=j+1$, we have from (4.6) $\Phi\left(t_{j+1}\right)=Y_{j+1}$ and

$$
\left\|\tilde{\Phi}\left(t_{j+1}\right)-Y_{j+1}\right\|_{\eta \xi} \leq \sigma(\Phi) e^{K_{\eta \xi} T}<\epsilon
$$

This implies that

$$
\mathbf{d}_{\eta \xi}\left(Y_{j+1}, R^{\left(t_{j+1}\right)}\left(X_{0}\right)\right)<\epsilon \text {. }
$$

Hence, we have

$$
Y_{j+1} \in R^{\left(t_{j+1}\right)}\left(X_{0}\right)+\epsilon B .
$$

Thus (4.4) holds for all $j=0,1,2 \cdots N$. This completes the proof of (i).

(ii) The values of $x \rightarrow P(x)(\eta, \xi)$ are now assumed to be convex. Thus Theorem (3.1) can be applied.

Let $\Phi(\cdot) \in S^{(T)}\left(X_{0}\right)$ such that its matrix elements

$<\eta, \Phi(\cdot) \xi>:=\Phi_{\eta \xi}(\cdot) \in C^{1}[0, T]$, for each pair of $\eta, \xi \in \mathbb{E}$.

By Theorem (3.1), any $\tilde{\Phi}(\cdot) \in S^{(T)}\left(X_{0}\right)$ can be approximated to any degree of accuracy by $\Phi(\cdot)$. Consequently, to prove $(4.2)$, it is sufficient to show that

$$
<\eta, \Phi(T) \xi>\in \lim _{N \rightarrow \infty} \inf \left(I+\frac{T}{N} P\right)^{N}\left(X_{0}\right)(\eta, \xi) .
$$

Denote by $Q$, the range of $\Phi(\cdot)$, i.e

$$
Q=\{x: x=\Phi(t), t \in[0, T]\}
$$

and choose $\theta>0$ so that

$$
Q+\theta B \subseteq \mathcal{N}
$$

Let $K_{\eta \xi}$ be Lipschitz constants for the map $P$ on $Q+\theta B$. For each integer $N, h=\frac{T}{N}, t_{j}=$ $j h, j=1,2 \cdots N$, define

$$
\varepsilon_{N, \eta \xi}=\sup _{j=0,1, \cdots N}\left|\frac{<\eta, \Phi\left(t_{j+1}\right) \xi>-<\eta, \Phi\left(t_{j}\right) \xi>}{h}-\frac{d}{d t}<\eta, \Phi(t) \xi>\right|_{t=t_{j}} \mid .
$$


Since $<\eta, \Phi(\cdot) \xi>$ is continuously differentiable on $[0, T], \varepsilon_{N, \eta \xi} \rightarrow 0$ as $N \rightarrow \infty$. Assuming that $N$ is large enough so that

$$
\varepsilon_{N, \eta \xi}<\frac{\theta K_{\eta \xi}}{e^{K_{\eta \xi} T}-1}
$$

then it can be shown that

$$
<\eta, \Phi(T) \xi>\epsilon\left(I+\frac{T}{N} P\right)^{N}\left(X_{0}\right)(\eta, \xi)+\frac{\varepsilon_{N, \eta \xi}}{K_{\eta \xi}}\left(e^{K_{\eta \xi} T}-1\right) B(\eta, \xi) .
$$

To prove (4.8), we proceed by letting $Y_{0}=X_{0}$ so that

$$
Y_{\eta \xi, 0}=<\eta, Y_{0} \xi>=<\eta, X_{0} \xi>
$$

and

$$
U_{\eta \xi, 0}=\frac{d}{d t}<\eta, \Phi(t) \xi>\left.\right|_{t=t_{0}}, \quad M_{0}=0 \in \mathbb{R}
$$

Having chosen

$$
Y_{\eta \xi, j}=<\eta, Y_{j} \xi>, \quad U_{\eta \xi, j}=<\eta, U_{j} \xi>
$$

for some $U_{j}, \quad Y_{j} \in \tilde{\mathcal{A}}$, let

$$
Y_{\eta \xi, j+1}=<\eta, Y_{j} \xi>+h<\eta, U_{j} \xi>
$$

where

$$
\begin{gathered}
Y_{j+1}=Y_{j}+h U_{j}, \\
U_{\eta \xi, j+1}=\operatorname{proj}\left(\frac{d}{d t}<\eta, \Phi(t) \xi>\left.\right|_{t=t_{j+1}}, P\left(Y_{j+1}\right)(\eta, \xi)\right)
\end{gathered}
$$

and

$$
M_{j+1}=\left(1+K_{\eta \xi} h\right) M_{j}+1 .
$$

We note that

$$
M_{j} \leq M_{j+1}
$$

for each $j$ and therefore by Lemma (2.2) ( with $R=1, S=1+K_{\eta \xi} h$ ) we have

$$
\left.M_{N}=\frac{1}{K_{\eta \xi} h}\left(\left(1+K_{\eta \xi} h\right)^{N}-1\right)\right) \leq \frac{1}{K_{\eta \xi} h}\left(e^{K_{\eta \xi} T}-1\right) .
$$

Inductively, suppose for $0 \leq j<N$, the estimate

$$
\left\|Y_{j}-\Phi\left(t_{j}\right)\right\|_{\eta \xi} \leq h \varepsilon_{N, \eta \xi} M_{j}
$$

holds. When $j=0,(4.10)$ is trivial as $Y_{0}=\Phi(0)$.

We have from (4.9) that

$$
h \varepsilon_{N, \eta \xi} M_{j} \leq \frac{\varepsilon_{N, \eta \xi}}{K_{\eta \xi}}\left(e^{K_{\eta \xi} T}-1\right) \leq \theta
$$


by the choice of $N$.

Hence (4.10) implies that

$$
Y_{j} \in Q+\theta B
$$

since $Q$ consists of elements in the range of $\Phi(\cdot)$ and

$$
B=\left\{x:\|x\|_{\eta \xi} \leq 1\right\} \subseteq \tilde{\mathcal{A}} .
$$

By the Lipschitz property of $P$ on $Q+\theta B$ and the choice of $U_{\eta \xi, j}$, we have

$$
\begin{aligned}
& \left|<\eta, U_{j} \xi>-\frac{d}{d t}<\eta, \Phi(t) \xi>\right|_{t=t_{j}} \mid \\
& =\rho\left(P\left(Y_{j}\right)(\eta, \xi), P\left(\Phi\left(t_{j}\right)\right)(\eta, \xi)\right. \\
& \leq K_{\eta \xi}\left\|Y_{j}-\Phi\left(t_{j}\right)\right\|_{\eta \xi} \leq K_{\eta \xi} h \varepsilon_{\eta \xi, N} M_{j}
\end{aligned}
$$

by (4.10).

Therefore,

$$
\begin{aligned}
& \left|<\eta, Y_{j+1} \xi>-<\eta, \Phi\left(t_{j+1}\right) \xi>\right| \\
& \leq\left|<\eta, Y_{j} \xi>-<\eta, \Phi\left(t_{j}\right) \xi>\right|+h\left|<\eta, U_{j} \xi>-\frac{d}{d t}<\eta, \Phi(t) \xi>\right|_{t j} \mid \\
& +\left|<\eta, \Phi\left(t_{j}\right) \xi>+h \frac{d}{d t}<\eta, \Phi(t) \xi>\right|_{t_{j}}-<\eta, \Phi\left(t_{j+1}\right) \xi>\mid \\
& \leq h \varepsilon_{N, \eta \xi} M_{j}+K_{\eta \xi} h^{2} \varepsilon_{N, \eta \xi} M_{j}+h \varepsilon_{N, \eta \xi}, \text { by }(4.10),(4.11), \text { and }(4.7) \\
& =h \varepsilon_{N, \eta \xi}\left[\left(1+K_{\eta \xi} h\right) M_{j}+1\right] \\
& =h \varepsilon_{N, \eta \xi} M_{j+1} .
\end{aligned}
$$

Hence

$$
\left\|Y_{j+1}-\Phi\left(t_{j+1}\right)\right\|_{\eta \xi} \leq h \varepsilon_{N, \eta \xi} M_{j+1} .
$$

The estimate (4.10) holds for $j+1$.

When $j=N,(4.10)$ combined with (4.9) leads to

$$
\begin{aligned}
& \left|<\eta, Y_{N} \xi>-<\eta, \Phi(T) \xi>\right|=\left\|Y_{N}-\Phi(T)\right\|_{\eta \xi} \\
& \leq \frac{\varepsilon_{N, \eta \xi}}{K_{\eta \xi}}\left(e^{K_{\eta \xi} T}-1\right)
\end{aligned}
$$

By the choice of $Y_{j}, \quad j=0,1,2 \cdots N,<\eta, Y_{N} \xi>$ lies in $P\left(I+\frac{T}{N} P\right)^{N}\left(X_{0}\right)(\eta, \xi)$ so that $(4.8)$ follows directly from (4.12).

By letting $N \rightarrow \infty$ in (4.8), the conclusion (4.2) follows.

The exponential formula is recorded in the next Corollary as an immediate consequence of Theorem 4.3. 
Corollary 4.4. Suppose that $\mathcal{N} \subseteq \tilde{\mathcal{A}}$ is open and $P$ is locally Lipschitzian on $\mathcal{N}$ with nonempty, compact and convex values. Then for any $X_{0} \in \mathcal{N}$, and $0 \leq T \leq \tilde{T}$, we have

$$
R^{(T)}\left(X_{0}\right)(\eta, \xi)=\lim _{N \rightarrow \infty}\left(I+\frac{T}{N} P\right)^{N}\left(X_{0}\right)(\eta, \xi) .
$$

The next Corollary indicates that the interval $[0, T]$ may be partitioned in an arbitrary manner provided that the width of the largest subinterval goes to zero. To this end, we need the following definitions.

If $D=\left\{t_{0}, t_{1}, \cdots, t_{N}\right\}$ is a patition of $[0, \mathrm{~T}]$ ( that is $t_{0}<t_{1}<t_{2} \cdots<t_{N}=T$ ), define

$$
|D|=\sup _{0 \leq j \leq N-1}\left|t_{j+1}-t_{j}\right|
$$

If $\left\{P_{\alpha \beta, j}\right\}_{j=1}^{N}$ is a collection of multifunctions $P_{\alpha \beta, j}: \tilde{\mathcal{A}} \rightarrow 2^{\tilde{\mathcal{A}}}$, define the multifunction product by

$$
\left(\Pi_{j=1}^{N} P_{\alpha \beta, j}\right)(x)=\left(P_{\alpha \beta, N} \circ P_{\alpha \beta, N-1} \circ \cdots P_{\alpha \beta, 1}\right)(x) .
$$

For $\eta, \xi \in \mathbb{E}$, this leads to the definition of the sesquilinear form:

$$
\left(\Pi_{j=1}^{N} P_{j}\right)(x)(\eta, \xi):=<\eta,\left(\Pi_{j=1}^{N} P_{\alpha \beta, j}\right)(x) \xi>
$$

Corollary 4.5. Suppose that $\mathcal{N}$ and $P$ are as in Corollary 4.4 and let $X_{0} \in \mathcal{N}, 0 \leq T<\tilde{T}$ and $\eta, \xi \in \mathbb{E}$. Then for any sequence of partitions $D_{k}=\left\{t_{0}^{k}, t_{1}^{k}, \cdots t_{N_{k}}^{k}\right\}$ of $[0, T]$ with $\left|D_{k}\right| \rightarrow 0$ as $k \rightarrow \infty$, we have

$$
R^{(T)}\left(X_{0}\right)(\eta, \xi)=\lim _{k \rightarrow \infty}\left(\Pi_{j=0}^{N_{k}-1}\left(I+\left(t_{j+1}^{k}-t_{j}^{k}\right) P\right)\left(X_{0}\right)(\eta, \xi) .\right.
$$

Proof: Follows similar steps as in the proof of Theorem 4.3 by replacing $h$ by $h_{j}^{k}:=t_{j+1}^{k}-t_{j}^{k}$.

\section{Approximations of Reachable Set for the QSDI}

Consider now the autonomous version of QSDI (1.2). Then the Euler approximation to the reachable set in the case of equally spaced partition $\left\{t_{i}\right\}$ can be written as follows

$$
\begin{aligned}
& R_{\eta \xi, 1}^{N}=(I+h P)\left(Y_{0}\right)(\eta, \xi) \\
& R_{\eta \xi, 2}^{N}=(I+h P)^{2}\left(Y_{0}\right)(\eta, \xi) \\
& R_{\eta \xi, i}^{N}=(I+h P)^{i}\left(Y_{0}\right)(\eta, \xi)
\end{aligned}
$$


where the power of $(I+h P)$ is that of composition of set valued map described in Section 4 . Then, by Theorem (4.3) and Corollary (4.4) $R_{\eta \xi, N}^{N}$ will converge to $R^{(T)}\left(X_{0}\right)(\eta, \xi)$ in the sense that

$$
R^{(T)}\left(X_{0}\right)(\eta, \xi)=\lim _{N \rightarrow \infty}\left(I+\frac{T}{N} P\right)^{N}\left(X_{0}\right)(\eta, \xi) .
$$

provided that the conditions of the Theorem are satisfied.

\section{ACKNOWLEDGEMENT}

I am grateful to Professor M. Virasoro, the Director of the Abdus Salam International Centre for Theoretical Physics, Trieste, Italy, for granting me a Visiting Research Fellowship in Mathematics for the year 2001. This work was undertaken while the Fellowship was being utilized at the Centre. I thank Professor A. O. Kuku of Mathematics Section, for his help and encouragement.

\section{REFERENCES}

[1] Aubin, J.P; Frankowska, H. : Set - Valued Analysis, Birkhauser (1990).

[2] Ayoola, E.O : On Numerical Procedures For Solving Lipschitzian Quantum Stochastic Differential Equations.Ph.D Thesis, University of Ibadan, Nigeria, 1998.

[3] Ayoola, E.O.: Solutions of Lipschizian quantum stochastic differential equations in a locally convex topological space, J. Science Research, 4 (1) (1998) 15-23.

[4] Ayoola, E.O. : Converging multistep methods for weak solutions of quantum stochastic differential equations, Stoch. Anal. Applic., 18(4), (2000) 525-554.

[5] Ayoola, E.O.: Construction of approximate attainability sets for Lipschitzian quantum stochastic differential inclusions, Stoch. Anal. Applic. 19(3), (2001) 461-471.

[6] Dontchev, A.L; Farkhi, E.M : Error estimates for discretized differential inclusions, Computing 41 (1989) 349-358.

[7] Dontchev, A; Lempio, F.: Difference methods for differential inclusions: A survey, SIAM Rev. 34(2), (1992) 263-294.

[8] Ekhaguere, G.O.S. : Lipschitzian quantum stochastic differential inclusions, Internat. J. Theoret. Phys. 31(11), (1992) 2003-2034.

[9] Ekhaguere, G.O.S.: Quantum stochastic differential inclusions of hypermaximal monotone type, Internat. J. Theoret. Phys. 34(3), (1995), 323-353.

[10] Ekhaguere, G.O.S.: Quantum stochastic evolutions. Internat. J. Theoret. Phys. 35(9), (1996), 1909-1946.

[11] Filipov, A.F,

(a) On the existence of solutions of multivalued differential equation Mat. Zametk, 10 (1971), 307-313.

(b) Classical Solutions of differential equations with multivalued right hand side, SIAM J. Control, 5 (1967) 609-621.

[12] Hudson, R.L; Parthasarathy, K.R.: Quantum Ito's formulae and stochastic evolutions, Commun. Math. Phys. 93, (1984) 301-324 
[13] Komarov, V.A. :Estimates of the attainability sets of differential inclusions. Mat. Zametki, 37(6), (1985) 916-925.

[14] V.A.Komarov, V.A; Pevchikh, K.E. : A method of approximating attainability sets for differential inclusions with a specified accuracy, Comput. Maths. Math. Phys, 31(1) (1991) 109-112.

[15] Phillips, E.R : An Introduction to Analysis and Integration Theory, Intext Educational Publishers, Scranton, Toronto, London, 1970.

[16] Rudin, W.:Real and Complex Analysis, McGraw-Hill, New York, 1974.

[17] Veliov, V.: Second order discrete approximation to linear differential inclusions, SIAM J. Num. Anal. 2, (1992) 439-451.

[18] Wolenski, Peter R. : A uniqueness theorem for differential inclusions, J.Differential Eq.,84 (1990), 165-182.

[19] Wolenski, Peter R. : The exponential formula for the reachable set of a Lipschitz differential inclusion, SIAM J. Control Optim. 28(5), (1990) 1148-1161. 